\title{
Phylogenetic relationships, virulence factors and Rep-PCR epidemiological analysis of $E_{\text {. coli from human sources }}$
}

\author{
Simona Caroppo',Vesselina Kroumova', Elena Grossini', Rosalia Ticozzi ${ }^{2}$,Alessandro Raimondi' , Giacomo Fortina' \\ I Laboratorio di Microbiologia e Virologia, Azienda Ospedaliera Universitaria Maggiore della Carità, Novara \\ 2 Dipartimento di Sanità Pubblica, Microbiologia, Virologia dell’Università degli Studi di Milano, Milano
}

Key words: Escherichia coli, Phylogenetic groups, Virulence factors, Rep-PCR

Gruppi filogenetici, fattori di virulenza ed analisi epidemiologica mediante Rep-PCR di ceppi di Escherichia coli isolati da materiali umani.

\section{SUMMARY}

The potential of Escherichia coli to cause of extra-intestinal infections was studied on a group of 94 clinical isolates. In this work, 32 E. coli isolates from urinary tract infections, 25 from bacteraemia, 12 from low respiratory tract infections, and 25 from the normal commensal flora were characterized for the phylogenetic type, the virulence factors (VFs) carriage and the Rep-PCR clonal composition.The B2 phylogenetic type was predominant among the urinary isolates (59\%), the B2 and D strains among the haematic isolates (32\% and $32 \%$ ). The A phylogenetic type was predominant among the commensal and the respiratory isolates ( $52 \%$ and $58 \%$ respectively). The distribution of the B2 type strains among the urinary isolates and of the D type strains among the faecal isolates was suggesting a urinary-origin for the B2 phylogenetic type isolates found in the blood and a direct faecal derivation for the haematic isolates with $D$ phylogenetic type. Twenty-nine VFs were analyzed.The B2 and D type strains carried a higher burden of VFs than the $\mathrm{A}$ and $\mathrm{BI}$ phylogenetic type strains (average of VFs/strain $=8$ vs 3). Some of the VFs were homogeneously distributed among the phylogenetic types (fimH, iutA, fyuA, traT). The PAI, papGIl, ibeA, KpsMTIII were exclusive of B2 and D phylogenetic type strains, while sfa/foc, focG, cnfl, hlyA and rfc were exclusively observed among the B2 type strains. The clustering analysis by Rep-PCR distinguished two groups of strains, the first including $96.77 \%$ of B2 and D type strains, while the second encompassing $91,5 \%$ of $A$ and $B I$ type strains.

\section{INTRODUZIONE}

Escherichia coli è un microrganismo che colonizza il tratto gastroenterico dell'uomo fin dalle prime ore di vita; raggiunge in tempi brevi un rapporto di relazione simbiotica con l'uomo conferendo all'intestino così colonizzato vantaggi nutritivi e di protezione verso altri microrganismi (29). In situazione di equilibrio con la microflora intestinale, E. coli non causa patologie se non nel caso di soggetti con sindrome di immunodepressione conclamata $(4,9)$ o nel caso di stipiti che, avendo acquisito specifici determinanti genetici di virulenza, siano diventati francamente enteropatogeni $(16,20)$.

Tuttavia, Escherichia coli è anche, nell'uomo, la più frequente causa di infezioni sostenute da batteri Gram-negativi in sede extra-intestinali quali cistiti, prostatiti, pielonefriti, batteriemie e meningiti neonatali $(12,13,24)$. Le differenze di patogenicità osservate nei ceppi di E. coli corrispondono spesso a differenze rilevanti nelle dimensioni e nell'organizzazione del genoma. Infatti, il ceppo comune commensale $E$. coli K12 ha un cromosoma di 4.639.221 bp, mentre il ceppo uropatogeno CFT073 ha un genoma di 5.231 .428 bp e il ceppo enteropatogeno EDL933 (O157:H7) ha un genoma di 5.528.445 bp.

Le differenze osservate riflettono la presenza di numerosi geni codificanti fattori di virulenza (FV) sul cromosoma di questi ceppi, spesso organizzati in grossi blocchi indicati come isole di patogenicità. La presenza delle isole di patogenicità facilita il trasferimento orizzontale contemporaneo di interi sistemi di geni di virulenza mediante plasmidi o batteriofagi. L'espressione di questi geni consente ad Escherichia coli di eludere i naturali meccanismi di difesa dell'organismo e di migrare dal suo habitat abituale ad altri distretti originando varie manifestazioni patologiche $(14,16,27)$.

L'associazione tra fattori di virulenza nelle isole di patogenicità determina il loro trasferimento in forma di blocchi di geni anziché di singoli geni. È probabile che questa situazione abbia evolutivamente contribuito alla formazione, e contribuisca attualmente al mantenimento, della struttura clonale che si osserva in questa specie. Si riconoscono attualmente quattro gruppi filogenetici principali di $E$. coli indicati come A, B1, B2, D. La maggior parte delle infezioni extrain- testinali da E. coli sono causate da ceppi di gruppo B2 e, in misura minore, di gruppo D. I ceppi di gruppo A e B1, al contrario, sono portatori di uno scarso patrimonio di geni di virulenza ed hanno un comportamento tipicamente commensale. L'approccio più recente per il riconoscimento dei diversi “patotipi” di E. coli consiste nell'individuare, attraverso le tecniche di biologia molecolare e l'amplificazione di specifici marcatori genici, la presenza di fattori di virulenza ed il tipo filogenetico di appartenenza. 94 ceppi di E. coli isolati da diversi materiali di provenienza umana sono stati sottoposti a questo tipo di indagine. La successiva analisi dei dati ottenuti ha permesso di valutare il grado di correlazione tra raggruppamenti basati su diversi aspetti: il pattern dei fattori di virulenza posseduti, il materiale biologico relativo all'isolamento ed i profili filogenetici $(7,11)$. I cluster formati secondo tali criteri sono stati inoltre confrontati con quelli ottenuti mediante la Rep-PCR per una valutazione dell'efficacia di questa nuova tecnica nel riconoscimento di specifici pattern genetici o di attitudini patogene.

\section{MATERIALI E METODI}

\section{Ceppi batterici}

Lo studio è stato condotto su 94 ceppi di $E$. coli, isolati da altrettanti pazienti. L'identificazione del microrganismo è stata eseguita con il sistema automatico Vitek autoMicrobic System (bioMérieux). I ceppi sono stati conservati a $-80^{\circ} \mathrm{C}$ in brodo Brain Heart Infusion Broth (BD) addizionato di glicerolo al $10 \%$.

I ceppi esaminati provenivano in 25 casi da sangue, in 32 da urine, in 12 da campioni respiratori costituiti da lavaggio bronco-alveolare (BAL) o aspirato endotracheale e in 25 casi da feci. Nel $70 \%$ dei casi si trattava di pazienti ricoverati, mentre il rimanente $30 \%$ era costituito da ceppi isolati da pazienti ambulatoriali.

\section{Estrazione del DNA}

I ceppi di $E$. coli, conservati a $-80^{\circ} \mathrm{C}$, sono stati coltivati su piastre di Agar sangue ed incubati per $18 \mathrm{~h}$ a $37^{\circ} \mathrm{C}$. In seguito, una singola colonia è stata risospesa in $500 \mu \mathrm{l}$ di acqua ultrapura DNAsi-free e sottoposta a shock termico $\left(94^{\circ} \mathrm{C}\right.$ per

\section{Corresponding author: Alessandro Raimondi}

Dipartimento di Sanità Pubblica, Microbiologia,Virologia dell'Università degli Studi Milano

Milano - Via Pascal, 36 - Tel.: 02 503I5086 - Fax: 02 503I5093

E-mail: alessandro.raimondi@unimi.it 
20') per estrarre il DNA da utilizzare come templato per le amplificazioni.

\section{Gruppi filogenetici}

L'analisi filogenetica è stata condotta utilizzando la metodica proposta da Clermont et al. (2). La metodica è basata su una PCR multiplex con tre coppie di primers: ChuA1/ ChuA2, YjaA1/ YjaA2, TspE4C2.1/TspE4C2.2. Ogni ceppo è stato attribuito ad uno dei 4 principali gruppi filogenetici indicati con le sigle A, B1, B2, D.

\section{Analisi genica dei fattori di virulenza (FV)}

I ceppi in esame sono stati tutti analizzati per rilevare la presenza di 29 marcatori significativi della presenza dei seguenti geni o associazioni di geni codificanti fattori di virulenza. In particolare sono stati analizzati: l'Isola di patogenicità denominata PAI (25), i geni codificanti per la produzione delle tossine cnf1, hlyA, cdtBa, cdtBs (28), i geni iutA, fyuA per la produzione di fattori connessi con il metabolismo dei siderofori (28), i geni fimH, pap $A H$, papC, papEF, nfaE, papGI, gafD, afa/dra, sfa/foc, pap GII, papGIII, focG, sfaS, bmaE coinvolti nella formazione di fimbrie o di adesine non fimbriali $(10,17)$, i geni kpsMT K1, kpsMT K5, kpsMTII, kpsMTIII per la formazione della capsula $(21,23,26)$, il gene rfc coinvolto nella produzione dell'LPS (19), il gene cvaC della colicina V (5), il gene ibeA collegato alla capacità di invadere l'endotelio cerebrale (19) ed il gene traT che conferisce aumentata siero resistenza $(9,11)$. Per l'amplificazione dei marcatori sopra elencati sono stati utilizzati i primers descritti da Johnson e Stell (11).

\section{Rep-PCR e analisi di clustering}

Novanta dei 94 ceppi di E. coli oggetto dello studio sono stati suddivisi in cluster mediante la tecnica di Rep-PCR (DIVERSILAB-bioMérieux, France) seguendo le istruzioni della ditta produttrice (6). Infine i prodotti di amplificazione ottenuti sono stati separati mediante tecnologia microfluidica LabChip ${ }^{\circledR}$. I profili elettroforetici dei diversi ceppi amplificati sono stati quindi confrontati da un software, fornito dalla ditta produttrice, che ha eseguito un'analisi di clustering secondo l'algoritmo di Dice, fornendo un dendrogramma dei ceppi analizzati ed una matrice di similarità.

\section{Analisi statistica}

I dati ottenuti sono stati analizzati per la ricerca di associazioni significative tra gruppo filogenetico, corredo di virulenza e sede d'isolamento, applicando il test esatto di Fisher per il confronto tra frequenze ed il test t di Student per il confronto fra medie.

\section{RISULTATI}

\section{Gruppi filogenetici}

Il gruppo di $E$. coli esaminato comprende 25 ceppi isolati da sangue, 25 da feci, 32 da urine e 12 da materiale respiratorio, tutti isolati da pazienti diversi. L'attribuzione dei singoli ceppi ai diversi gruppi filogenetici ha evidenziato, nella popolazione allo studio, la presenza di 34 ceppi con genotipo A, 31 ceppi con genotipo B2, 13 di genotipo B1 e 17 di genotipo D.

La tabella 1 riporta nel dettaglio, per ciascun gruppo filogenetico, il numero dei ceppi isolati da ogni singolo materiale di provenienza accompagnato dalla frequenza calcolata con- siderando uguale a cento al numero totale di ceppi del gruppo filogenetico stesso.

E evidente da questa analisi come il 61\% dei ceppi di gruppo B2 risultino isolati da urine, il 50\% dei ceppi di gruppo D siano stati isolati da sangue mentre $38 \%$ dei ceppi di gruppo A e $47 \%$ di quelli di gruppo B1 provenga dalle feci.

Nella tabella 2, la stessa distribuzione numerica è stata analizzata secondo un criterio ortogonale al precedente allo scopo di evidenziare la frequenza relativa con cui i quattro tipi filogenetici compaiono tra i ceppi isolati da ogni specifica sede che sono stati considerati, ogni volta, uguali a cento. Si può osservare che i ceppi isolati da urine sono, coerentemente alla precedente distribuzione, appartenenti al gruppo filogenetico B2 nel 59\% dei casi $(P<0.001)$, mentre i microrganismi isolati da sangue appartengono per un terzo al gruppo B2 e per un terzo al gruppo D; il tipo filogenetico A rappresenta il $52 \%$ dei casi tra gli isolati da feci $(P<0.05)$ e risulta prevalente anche tra i ceppi isolati da materiale respiratorio (58\%) che costituiscono invero una componente esigua del campione analizzato.

È interessante inoltre notare la frequenza particolarmente bassa (associazione negativa) dei ceppi di gruppo $\mathrm{D}$ nelle urine, che è pari al $3 \%(P<0.01)$, e di gruppo $\mathrm{B} 2$ nelle feci, pari al $4 \%(P<0.001)$.

\section{Distribuzione dei fattori di virulenza}

La distribuzione dei geni codificanti i fattori di virulenza $(\mathrm{FV})$ in esame è stata analizzata, nella tabella 3 , in relazione al gruppo filogenetico di appartenenza ed alla provenienza dei ceppi.

Il numero medio di FV/ceppo risulta molto maggiore tra i ceppi di gruppo filogenetico B2 e D rispetto a quelli di gruppo A e B1. Relativamente alla sede di isolamento, la presenza di FV è più alta nei ceppi urinari rispetto agli isolati da sangue e da materiale respiratorio ed è nettamente inferiore negli isolati fecali.

Considerati singolarmente, alcuni FV risultano distribuiti con grande omogeneità indipendentemente dal gruppo filogenetico o dalla provenienza (fimH, iutA, traT). Alcuni FV sono stati osservati esclusivamente nel gruppo filogenetico B2 (sfa/foc, papGIII, focG, cnf1, hlyA), esclusivamente o prevalentemente nei gruppi B2 e D (PAI, papAH, papC, papEF, papGII, K5, kpsMTII, kpsMTIII). Due soli FV sono prevalenti nei gruppi B1 ed A (bmaE, cvaC).

Nei ceppi di provenienza urinaria ed ematica alcuni FV risultano nettamente prevalenti (PAI, sfa/foc, papGIII, focG, cnf1, hlyA, kpsMTIII, $r f c$ ), mentre nei campioni dalle vie respiratorie si osserva una frequenza più alta di papGII e di cvaC. Sei tra i 29 fattori di virulenza saggiati, (nfaE, papGI, gafD, $c d t B a, c d t B s, a f a / d r a)$ non sono mai risultati presenti.

\section{Rep-PCR e analisi di clustering}

L'esame di 90 dei 94 ceppi di E. coli in studio mediante Rep-PCR e la successiva analisi di clustering hanno evidenziato 2 grossi raggruppamenti, che contengono 78 dei 90 campioni saggiati (figura I). Il cluster superiore (linea tratteggiata) raggruppa 31 ceppi, il 96.77\% dei quali appartiene ai gruppi filogenetici B2 o D. Il cluster inferiore (linea intera) raggruppa 59 ceppi, di cui il $91.5 \%$ di gruppo A e B1. I rimanenti 12 ceppi mostrano un'eterogeneità che non consente clusterizzazioni significative.

Tabella I. Distribuzione e frequenza di isolamento dei 94 ceppi di E. coli esaminati: ripartizione di ciascun filogruppo tra le diverse sedi di isolamento.

\begin{tabular}{|c|c|c|c|c|c|c|c|c|c|}
\hline (2) & & & VUM & ROE E & ISO & ATI D & & & \\
\hline GRUPPO FILOGENETICO & SAI & GUE & & & & NE & MATEF & LE RESP. & TOT. (\%) \\
\hline & $\mathbf{N}$ & (\%) & $\mathbf{N}$ & (\%) & $\mathbf{N}$ & $(\%)$ & $\mathbf{N}$ & $(\%)$ & \\
\hline A & 5 & (15) & 13 & (38) & 9 & (26) & 7 & (2I) & $34(100)$ \\
\hline B I & 4 & (30) & 6 & (47) & 3 & (23) & 0 & (0) & $13(100)$ \\
\hline B2 & 8 & (25) & $\mathrm{I}$ & (3) & 19 & (62) & 3 & (10) & $3 I(100)$ \\
\hline D & 8 & $(50)$ & 5 & (31) & $\mathrm{I}$ & (6) & 2 & $(13)$ & $16(100)$ \\
\hline TOT. & 25 & & 25 & & 32 & & 12 & & 94 \\
\hline
\end{tabular}


Tabella 2. Distribuzione e frequenza di isolamento dei 94 ceppi di E. coli esaminati: frequenza di rilevazione dei diversi filogruppi in rapporto alla sede d'isolamento.

\begin{tabular}{|c|c|c|c|c|c|}
\hline sede d'isolamento & $\mathbf{A}$ & B I & B2 & $\mathbf{D}$ & $\mathbf{n}^{\circ}$ ceppi (\%) \\
\hline sangue & 20 & 16 & 32 & 32 & $25(100)$ \\
\hline feci & 52 & 24 & 4 & 20 & $25(100)$ \\
\hline urine & 28 & 9 & 59 & 3 & $32(100)$ \\
\hline materiale respiratorio & 58 & 0 & 25 & 17 & $12(100)$ \\
\hline
\end{tabular}

Tabella 3. Frequenza di distribuzione dei fattori di patogenicità nei ceppi di E. coli in rapporto al tipo filogenetico ed alla sede d'isolamento.

Frequenza \% di distribuzione di Fattori di Virulenza rispetto

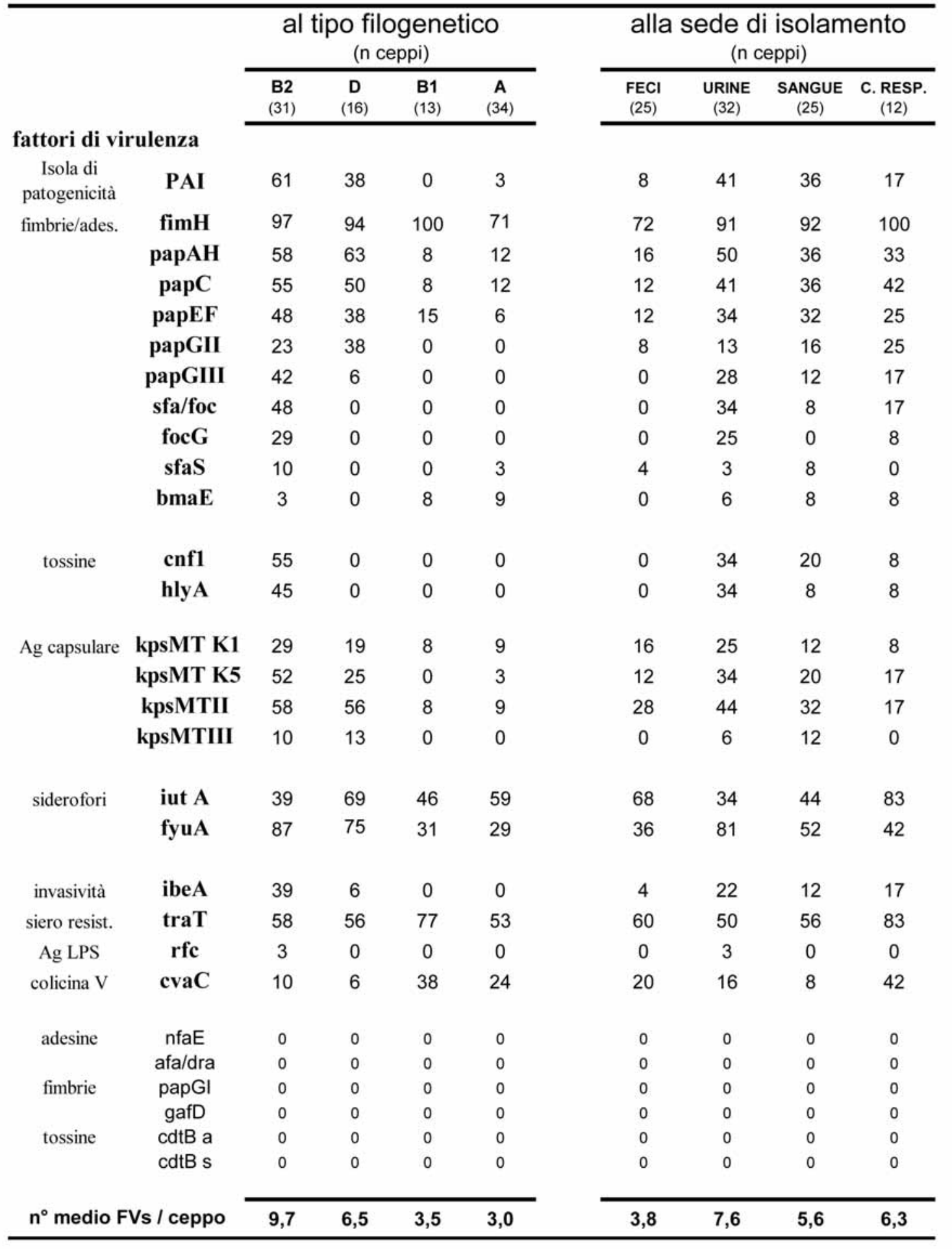




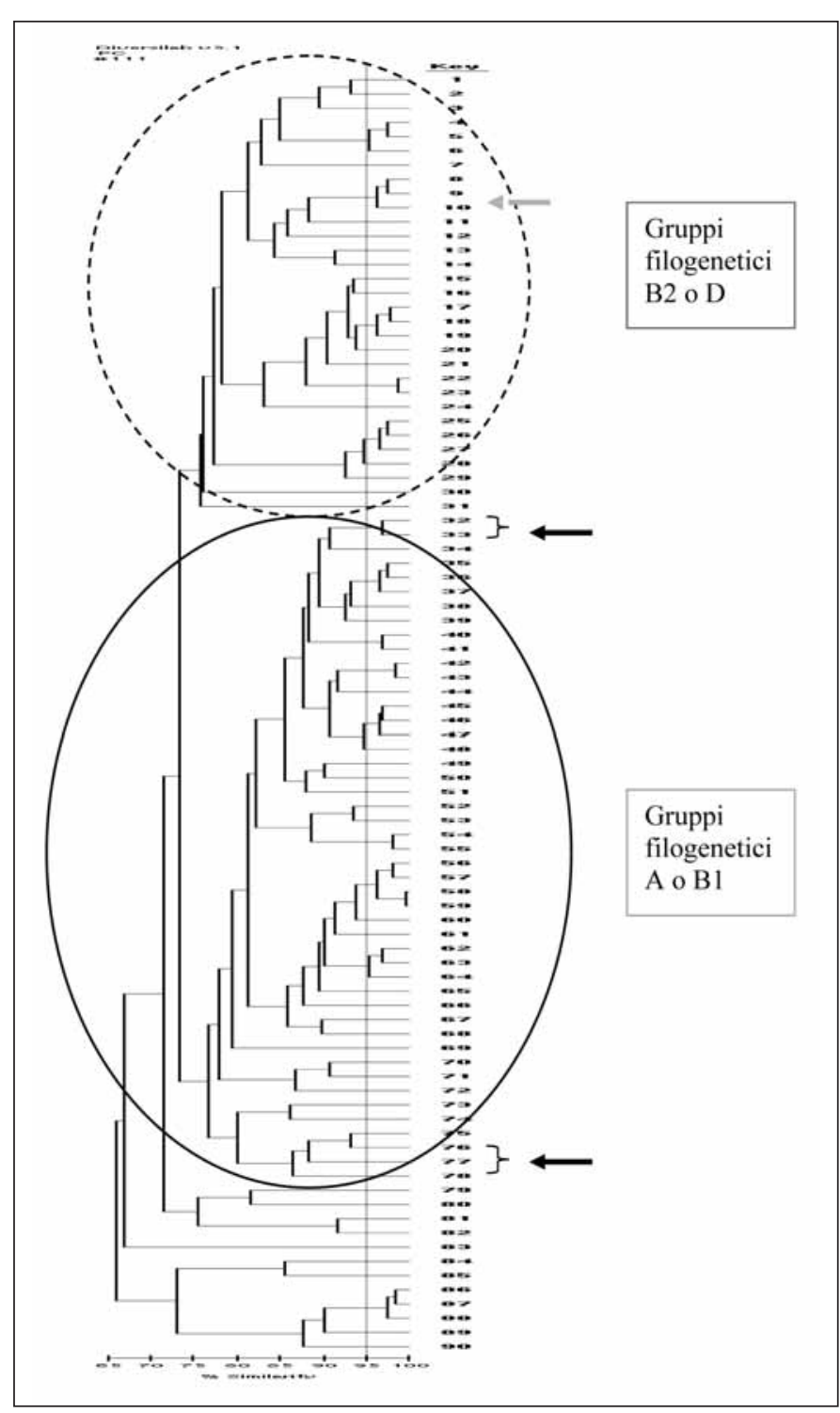

Figura I. Analisi di clustering di 90 ceppi di E. coli esaminati mediante Rep-PCR. Gruppo filogeneticoB2 o D Gruppo filogenetico A.

\section{DISCUSSIONE}

In questo studio sono stati esaminati 94 ceppi di E. coli isolati da materiale proveniente da diverse sedi corporee (intestino, circolo sanguigno, apparato urinario e vie respiratorie) di altrettanti pazienti.

I ceppi sono stati suddivisi sulla base dell'appartenenza ad uno dei quattro gruppi filogenetici, A, B1, B2 e D. Questa distribuzione è stata poi comparata con la suddivisione clonale ottenuta mediante la tecnica Rep-PCR. I ceppi sono stati inoltre esaminati per la presenza di 29 geni per fattori di virulenza (FV) extraintestinali.

All'analisi filogenetica, i ceppi del campione analizzato sono risultati appartenere per un terzo al gruppo filogenetico A, per un terzo al gruppo filogenetico B2 ed i rimanenti ugualmente distruibuiti tra i gruppi B1 e D.

I ceppi di gruppo filogenetico B2 e D sono stati complessivamente ritrovati nel 64\% degli isolati da sangue, nel 62\% degli isolati da urine e nel $42 \%$ dei campioni respiratori. Gli stessi gruppi erano complessivamente presenti solo nel $24 \%$ dei campioni fecali che invece ospitano nel 76\% dei casi ceppi di gruppo filogenetico A oppure B1. Questi dati confermano quelli di letteratura, che descrivono i gruppi filogenetici B2 e $\mathrm{D}$ come i più frequentemente responsabili di patologia in sede extraintestinale ed i tipi A e B1 come commensali intestinali $(1,7,11,15,18)$.
È interessante osservare inoltre che i gruppi filogenetici B2 e D, che compaiono con identica frequenza tra i ceppi di isolamento ematico (32\%), hanno una frequenza di distribuzione molto diversa, ed anzi opposta, tra i ceppi di isolamento urinario (B2= 59\%, $\mathrm{D}=3 \%$ ) rispetto a quelli fecali (B2=4\%, $\mathrm{D}=20 \%)$.

La prevalenza dei ceppi di gruppo filogenetico B2 tra gli isolamenti urinari e dei ceppi di gruppo D tra i fecali potrebbe suggerire una diversa derivazione dei ceppi di isolamento ematico appartenenti a questi gruppi filogenetici. I dati sembrano infatti indicare un passaggio diretto dall'intestino al sangue nel caso di ceppi di tipo D, mentre per i ceppi di tipo B2 sembra più probabile un passaggio ed una selezione attraverso l'apparato urinario prima della fase batteriemica. Curiosamente, i ceppi isolati da sede respiratoria sono ugualmente suddivisi tra gruppi filogenetici potenzialmente più patogeni (42\%) e potenzialmente commensali (58\%). Il numero limitato di questi campioni non consente alcuna conclusione in merito; da un punto di vista puramente speculativo è opportuno tuttavia ricordare che le infezioni respiratorie sostenute da enterobatteri si verificano prevalentemente in soggetti anziani o immunitariamente deboli, situazione che facilita l'aggressione anche da parte di ceppi non particolarmente virulenti. Relativamente ai gruppi filogenetici isolati nei campioni uricnari si osserva una netta prevalenza (59\%) di ceppi appartenenti al gruppo B2 come rilevato anche da Piatti, Carattoli ed altri $(3,8,22)$.

Analizzando la presenza di fattori di virulenza extraintestinali, è emerso quanto segue:

coerentemente con la maggior attitudine dei ceppi di gruppo B2 e D a causare infezione in sede urinaria ed ematica, è stata evidenziata una maggior frequenza di fattori di virulenza nei ceppi appartenenti a questi gruppi rispetto ai gruppi A e B1 (frequenza media = 8 vs $3 ; P<0.001)$.

Inoltre, considerata la frequente associazione di fattori di virulenza in "isole di patogenicità", è presumibile che la prevalenza di fattori di virulenza nei gruppi filogenetici B2 e D sia anche maggiore di quanto evidenziato attraverso il ridotto numero di fattori esaminati.

Alcuni geni per FV, come l'adesina delle fimbrie di tipo I, fimH, il fattore di resistenza sierica traT, l'aerobactina iutA ed in misura minore la yersinobactina fyuA risultano uniformemente distribuiti tra ceppi di diverso gruppo filogenetico e sedi di isolamento, suggerendo una colonizzazione evolutivamente precoce ed in posizione stabile del genoma della specie E. coli. Relativamente a fyuA, inoltre, i nostri risultati concordano con l'alta prevalenza di questo marcatore riscontrata tra i ceppi uropatogeni da altri autori (11), ma non sembrano confermare la possibilità del suo utilizzo come indicatore potenziale di attitudine emoinvasiva.

Alcuni elementi di patogenicità, (in particolare i marcatori per i geni fimbriali papAH, papC, papEF, papGII/GIII, i marcatori capsulari kpsMTK1/K5/II/III, e l'isola di patogenicità $\mathrm{PAI})$ sono risultati più frequentemente espressi dai gruppi B2 e D rispetto ai gruppi A e B1. Altri appaiono, dal nostro studio, come tipici dei ceppi di tipo B2 (le adesine $s f a / f o c$ e foc $G$, il fattore necrotizzante di tipo 1 cnf1 e l'emolisina hlyA).

Nessun marcatore risulta associato in modo assoluto all'isolamento da sangue o da feci, tuttavia i marcatori dei geni sfa/foc, focG, hly e papGIII sembrano fortemente associati ad urovirulenza, mentre l'isola di patogenicità PAI ed il marcatore del gene cnf1 sembrano associabili sia ad urovirulenza che a batteriemia. Curiosamente cvaC, marcatore della presenza del plasmide ColV e della produzione di colicina V, compare con frequenza nettamente più alta nei gruppi filoge- 
netici A e B1 e nei ceppi isolati da campioni respiratori. Alcuni marcatori riportati, anche se con bassa frequenza, da altri autori sono qui completamente assenti.

L'analisi di clustering mediante la tecnica di Rep-PCR ha evidenziato la suddivisione della popolazione in esame in due grandi clusters, che raggruppano la quasi totalità dei ceppi appartenenti rispettivamente ai gruppi B2 e D ed ai gruppi A e B1. Fanno eccezione, rispetto a questa suddivisione, 6 ceppi di $E$. coli che sembrano rientrare in un cluster non congruente con il loro gruppo filogenetico (figura I). In un caso l'alto numero di FV posseduti è congruente con il cluster di appartenenza ma non con il tipo filogenetico (il ceppo posizionato nel cluster superiore nella figura I possiede, coerentemente al cluster di appartenenza, numerosi fattori di virulenza ma appartiene al gruppo filogenetico A). In tre ceppi di gruppo filogenetico B2, posizionati nel cluster inferiore (figura I), il numero di FV è congruente con il gruppo filogenetico ma non con il cluster. Infine, per i due ceppi di E. coli posizionati nella figura I in posizione intermedia fra $\mathrm{i}$ due cluster, la metodica Rep-PCR sembra offrire una bassa capacità discriminante. Per questi ceppi manca, d'altra parte, coerenza anche rispetto alla relazione tra FV e gruppo filogenetico, appartenendo al gruppo filogenetico B2 ma possedendo un numero molto ridotto di fattori di patogenicità (1 e 2 FV rispettivamente).

I dati emersi confermano in linea generale la relazione tra gruppo filogenetico di appartenenza, cluster Rep-PCR e potenziale patogenicità dei ceppi di $E$. coli.

La diversa organizzazione genetica presente all'interno di questi gruppi viene riconosciuta distintamente, pur attraverso elementi diversi, da entrambe le metodiche con efficienza pressoché identica. Esiste evidentemente, all'interno dell'organizzazione del genoma, una relazione stretta tra la presenza di geni per particolari FV, i marcatori molecolari utilizzati per il riconoscimento dei gruppi filogenetici e la distribuzione delle sequenze Rep all'interno del cromosoma batterico, tradotta dalla tecnica Rep-PCR in un conseguente profilo elettroforetico .

Molte delle indagini svolte in precedenza sui FV hanno analizzato popolazioni di E. coli di derivazione omogenea, prevalentemente uropatogene. La nostra analisi della distribuzione dei tipi filogenetici e dei FV ha coinvolto ceppi di derivazione più varia e questo ha consentito di formulare ipotesi sulle le vie di diffusione delle infezioni extraintestinali di origine endogena. Pur nei limiti imposti dal basso numero dei ceppi analizzati, l'analisi del nostro campione sembra suggerire che il passaggio dall'intestino al sangue segua un percorso diretto per i ceppi di gruppo D, mentre quelli di gruppo B2 sembrano prima transitare e selezionarsi attraverso l'apparato urinario.

Come emerso in precedenti studi, l'analisi della distribuzione dei FV mirava anche ad individuare le possibili correlazioni tra questi ed il quadro patologico di provenienza e quindi a verificare la possibilità di usare alcuni FV come markers prognostici negativi $(4,14,16)$.

Sebbene non conclusivi, alcuni aspetti emersi dallo studio possono effettivamente contribuire a questo obbiettivo. Tra questi, la conferma della presenza dell'operone sfa/foc nei soli ceppi di filotipo B2, il rilevamento esclusivo (ed indicativo della presenza dell’isola di patogenicità PAI II ${ }_{j 96}$ ), di cnf1 ed hlyA associato a papGIII in ceppi di filotipo B2 e la bassa frequenza $(<1 / 25)$ degli stessi marcatori tra la popolazione commensale intestinale tendono ad enfatizzare il ruolo di queste componenti nella invasività extraintestinale di E. coli.

\section{BIBLIOGRAFIA}

1. Boyd EF, Hartl DL. Chromosal regions specific to pathogenic isolates of Escherichia coli have a phylogenetically clustered distribution. J Bacteriol, 1998; 180: 1159-65.

2. Clermont O, Bonacorsi S, Bingen E. Rapid and simple determination of the Escherichia coli phylogenetic group. Appl Environ Microbial, 2000; 66: 4555-8.

3. Carattoli A, Garcia-Fernandez A, Varesi P, et al. Molecular epidemiology of Escherichia coli producine estended-spectrum ß-lactameses isolated in Rome, Italy. J Clin Microbiol 2008; 46: 103-8.

4. Dobrindt U, Hacker J. Targeting virulence traits: potential strategies to combat extraintestinal pathogenic E. coli infections. Current Opinion in Microbiology, 2008; 11: 409-13.

5. Gilson L, Mahanty H.K, Kolter R. Four plasmid genes are required for colicin V syntesis, export and immunity. J Bacteriol, 1987; 169: 2466-70.

6. Healy M, Bittner T, Lising M, et al. Microbial DNA typing by automated rep-PCR. J Clin Microbiol 2005; 43: 199-207.

7. Herzer PJ, Inouye S, Inouye M, Wittam TS. Phylogenetic distribution of branched RNS-linked multicopy single-stranded DNA among natural isolates of Escherichia coli. J Bacteriol 1990; 172: 6175-81.

8. Houndouin V, Bonacorsi S, Bidet P, Bingen-Bidois M, Barraud D, Bingen E. Phylogenetic background and carriage of pathogenicity island-like domains in relation to antibiotic resistance profiles among Escherichia coli urosepsis isolates. J Antimicrob Chemother 2006; 58: 748-51.

9. Johnson JR. Virulence factors in Escherichia coli urinary tract infection. Clin Microbiol Rev 1991; 4: 80-128.

10. Johnson JR, Brown JJ. A novel multiply-primed polymerase chain reaction assay for identification of variant pap $G$ genes encoding the Gal (al-4) Galbinding PapG adhesion of Escherichia coli. J Infect Dis 1996; 173: 920-6.

11. Johnson JR, Stell AL. Extended virulence genotypes of Escherichia coli from patients with urosepsis in relation to phylogeny and host compromise. J Infect Dis 2000; 181: 261-72.

12. Johnson JR. Microbial virulence determinants and the pathogenesis of urinary tract infection. Infect Dis Clin North Am 2003; 17: 261-78.

13. Johnson JR, Gajewski A, Lesse AJ, Russo TA. Extraintestinal pathogenic Escherichia coli as a cause of invasive non-urinary infections. J Clin Microbiol 2003; 41: 5798-802.

14. Johnson JR. Virulence factors in Escherichia coli. J Clin Microbiol 2005; 43 6221-2.

15. Johnson JR, Owens KL, Clabots CR, Weissman SJ, Cannon SB Phylogenetic relationships among clonal group of extraintestinal pathogenic Escherichia coli as assessed by multi-locus sequence analysis. Microb Infect 2006; 8: 1702-13.

16. Kaper JB, Nataro JP, Mobley HLT. Pathogenic Escherichia coli. Nature Reviews Microbilogy 2004; 2: 123-40.

17. Le Bouguenec C, Archambaud M, Labigne A. Rapid and specific detection of the pap, afa and sfa adhesion-encodifing operons in uropathogenic Escherichia coli strains by polymerase chain reaction. J Clin Microbiol 1992; 30: 1189-93.

18. Lecointre G, Rachdi L, Darlu P, Denamur E. Escherichia coli molecular phylogeny using the incongruence lenght difference test. Mol Biol Evol 1998; 15: 1685-95.

19. Lukomoski S, Hull RA, Hull SI. Identification of the $O$ antigen polymerase $(r f c)$ gene in Escherichia coli $\mathrm{O} 4$ by insertional mutagenesis using a non polar chloramphenicol resistance cassette. J Bacteriol 1996; 178: 240-7.

20. Nataro JP, Kaper JB. Diarrheagenic Escherichia coli. Clin Microbiol Rev 1998; 11: 142-201.

21. Pavelka MS, Wright LF, Silver RP. Identification of two genes kpsM and $k p s T$, in region 3 of the polysialic acid gene cluster of Escherichia coli K1. J Bacteriol 1991; 173: 4603-10.

22. Piatti G, Mannini A, Balistreri M, Schito AM. Virulence factors in urinary Escherichia coli strains: phylogenetic background and quinolone and fluorochinolone resistance. J Clin Microbiol 2008; 46: 480-7.

23. Russo TA, Wenderoth S, Carlino UB, Merrick JM, Lesse AJ. Identification genomic organization, and analysis of the group III capsular polysaccharide genes $k p s D, k p s M$, kpsT, and kpsE fro an extraintestinal isolate of Escherichia coli. J Bacteriol 1998; 180: 338-49.

24. Russo TA, Johnson JR. Medical and economic impact of extraintestinal infection due to Escherichia coli: an overlooked epidemic. Microbes Infect 2003; 5: 449-56.

25. Schimdt H, Hensel M. Pathogenicity islands in bacterial pathogenesis. Clin Microbiol Rev 2004; 17: 14-56.

26. Smith AN, Boulnois GJ, Roberts IS. Molecular analysis of the Escherichia coli K3 kps locus: identification and characterization of an inner-membrane capsular polysaccharide transport system. Mol Microbiol 1990; 4: 1863-9.

27. Wiles TJ, Kulesus RR, Mulvey MA. Origins and virulence mechanisms of uropathogenic Escherichia coli. Experimental and molecular pathology, 2008; 85: 11-9.

28. Yamamoto S, Terai A, Yuri K, Kurazono H, Takeda Y, Yoshida O. Detection of urovirulence factors in Escherichia coli by multiplex polymerase chain reaction. FEMS Immunol Med Microbiol 1995; 12: 85-90.

29. Yan F, Polk DB. Commensal bacteria in the gut: learning who our friends are Current Opinions Gastroenterology, 2004; 20: 565-71. 